\title{
Symmetry Breaking in Tournaments
}

\author{
Antoni Lozano* \\ Research Group on Combinatorics, Graph Theory, and Applications \\ Universitat Politècnica de Catalunya \\ Catalonia, Spain \\ antoni@lsi.upc.edu
}

Submitted: Nov 11, 2010; Accepted: Mar 15, 2013; Published: Mar 24, 2013

Mathematics Subject Classifications: 05C12; 05C20

\begin{abstract}
We provide upper bounds for the determining number and the metric dimension of tournaments. A set of vertices $S \subseteq V(T)$ is a determining set for a tournament $T$ if every nontrivial automorphism of $T$ moves at least one vertex of $S$, while $S$ is a resolving set for $T$ if every two distinct vertices in $T$ have different distances to some vertex in $S$. We show that the minimum size of a determining set for an order $n$ tournament (its determining number) is bounded by $\lfloor n / 3\rfloor$, while the minimum size of a resolving set for an order $n$ strong tournament (its metric dimension) is bounded by $\lfloor n / 2\rfloor$. Both bounds are optimal.
\end{abstract}

Keywords: tournament graphs, determining number, metric dimension

\section{Introduction}

The idea of somehow fixing the vertices of a graph in order to destroy all its nontrivial automorphisms has captured the attention of reserchers in recent years, leading to theoretical results for different graph families (trees [8], Kneser graphs [2], digraphs [7], wheels [17], or hypercubes [6]) and to applications in areas such as robotics [13] or chemistry [5].

One can distinguish vertices in a graph by considering their distances to a given subset of fixed vertices: If every two distinct vertices of the graph have different distances to some vertex in the chosen subset, no nontrivial automorphism can be left. In a more nonconstructive approach, one can just fix a given set of vertices so that no nontrivial automorphism is possible in the graph without caring whether it is due to distances or not. The set fixed in the first approach is known as a resolving set $[16,10]$, while the

\footnotetext{
*Supported by MEC and European Regional Development Fund under project MTM2011-28800-C02-
} 01 and bu MICINN project TIN2011-27479-C04-03. 
set fixed in the second one has been called fixing set [8] and determining set [4]. In both cases, it is desirable to find a set of minimum size which "fixes" or "destroys" all nontrivial automorphisms in a graph, making the automorphism group of the resulting structure - say, a labeled graph - trivial. This way, the sizes of the smallest determining and resolving sets for a graph can be seen as two parameters on its degree of symmetry.

Additional ways for fixing the vertices in a graph have been considered, for example, by Harary [9] and also by Albertson and Collins [3] who introduced the term symmetry breaking; this term will be used here to refer to the preceeding approaches as a whole, while the associated parameters to be minimized will be called symmetry parameters.

Tournament graphs have been extensively studied (for classical references, see $[11,14]$ ). Some of their well-known properties (for example, the fact that their automorphism groups have odd order and, hence, are solvable) may help to get stronger conclusions on symmetry breaking in tournaments than in general directed graphs. In this paper, we consider determining and resolving sets for tournaments and prove the existence of optimal upper bounds for their minimum sizes amounting to constant fractions of their order: $\frac{1}{3}$ for determining sets, and $\frac{1}{2}$ for resolving sets of strong tournaments.

\section{$1.1 \quad$ Tournaments}

If $D$ is a directed graph (digraph for short) $V(D)$ and $A(D)$ will denote the vertex set and the $\operatorname{arc}$ set of $D$. Given two distinct vertices $u, v$ of $D$, we will write $u \in D$ instead of $u \in V(D)$ and $u v \in A(D)$ rather than $(u, v) \in A(D)$. The notation $d_{D}(x, y)$ (or just $d(u, v)$ when $D$ is understood) stands for the directed distance from $u$ to $v$ in $D$, that is, the length of the shortest directed path from $u$ to $v$ in $D$.

A tournament models the outcome of a competition where every player plays against each other in a 2-player match. Formally, a digraph $T$ is a tournament if between every pair of distinct vertices $u, v \in T$, we have $u v \in T$ or $v u \in T$ but not both. For any tournament $T$, define the following subsets of its vertices for any $u, v \in T$ :

- $T_{u \rightarrow}=\{w \in T \mid u w \in T\}$

- $T_{\rightarrow u}=\{w \in T \mid w u \in T\}$

- $T_{u v \rightarrow}=T_{u \rightarrow} \cap T_{v \rightarrow}$

- $T_{u \rightarrow j}=T_{u \rightarrow} \cap T_{\rightarrow v}$

- $T_{\rightarrow u v}=T_{\rightarrow u} \cap T_{\rightarrow v}$

The indegree of a vertex $u$ is $\left|T_{\rightarrow u}\right|$ while its outdegree is $\left|T_{u \rightarrow}\right|$. A tournament $T$ is regular if the indegree (and, consequently, the outdegree) of all vertices is the same. In the case of a regular tournament $T$ of order $2 n+1$, it is easy to see that $\left|T_{u \rightarrow}\right|=\left|T_{\rightarrow u}\right|=n$ for every vertex $u \in T$.

A tournament $T$ is transitive if its vertices can be numbered from 1 up to $n$ in such a way that every arc $i j$ in $T$ satisfies $i<j$. Therefore, there is a unique transitive 
tournament of order $n$ up to isomophism, which is denoted by $T T_{n}$. Since the existence of a directed 3-cycle - which we will denote by $C_{3}$ - contradicts the condition in the definition of $T T_{n}$, a characterization of transitive tournaments, then, is precisely that they do not contain the subtournament $C_{3}$.

A tournament is strong if all directed distances between vertices are defined. It is obvious that while $T T_{n}$ is not strong, the almost transitive tournament of order $n \geqslant 3$, $T T_{n}^{*}$, consisting of a copy of $T T_{n}$ with the arc from 1 to $n$ reversed is strong.

\subsection{Symmetry Parameters}

Given a digraph $D$, we denote by $\Gamma(D)$ the automorphism group of $D$ and by $S_{1}$ the trivial group. We note the well-known fact that every automorphism in $\Gamma(D)$ is an isometry, that is, for any $u, v \in D$ and $\phi \in \Gamma(D), d(u, v)=d(\phi(u), \phi(v))$. We say that an automorphism $\phi$ fixes a vertex $u$ if $\phi(u)=u$ and that it fixes a set $S \subseteq V(D)$ if it fixes every $u \in S$. The set of automorphisms of $D$ that fix the set $S$ is a subgroup of $\Gamma(D)$ called pointwise stabilizer of $S$ in $D$, and we denote it by $\operatorname{Stab}_{\Gamma(D)}(S)$ (or just $\operatorname{Stab}(S)$ when $D$ is understood). Given a vertex $u$ of $D$, the set $\mathcal{O}_{D}(u)=\{\phi(u) \mid \phi \in \Gamma(D)\}$ is called the orbit of $u$ in $D$. Orbits of $D$ induce a partition in the set of vertices. Note that no automorphism can move a vertex from an orbit to another.

Definition 1. A set $S \subseteq V(D)$ for which $\operatorname{Stab}_{\Gamma(D)}(S)=S_{1}$ is called a determining set of $D$, and the minimum cardinality of a determining set for $D$, denoted by $\operatorname{Det}(D)$, is called determining number of $D$.

A digraph with no nontrivial automorphisms is called rigid. Determining sets (also known as fixing sets) have been introduced independently by Erwin and Harary [8] and by Boutin [4]. As a simple example, a determining set for $C_{3}$ is any set containing one vertex, while the empty set is a determining set for $T T_{k}$, for any $k$, since any transitive tournament is rigid. Also note that any set with $n-2$ vertices of a tournament of order $n$ is a determining set, since no automorphism can exchange the remaining two vertices (tournaments do not have involutions).

In a different approach introduced separately by Harary and Melter [10], and by Slater [16], the vertices in a graph can be distinguished according to their distances to a given subset of vertices.

Definition 2. We say that a vertex $u$ of a digraph $D$ resolves a pair of vertices $v, w \in D$ if $d(v, u) \neq d(w, u)$. We also say that $S \subseteq V(D)$ is a resolving set for $D$ if every pair of vertices in $D$ can be resolved by some vertex in $S$. The minimum size of a resolving set for $D$ is referred to as the metric dimension of $D$, and is denoted by $\operatorname{Dim}(D)$.

While the metric dimension of a digraph does not need to be defined, the situation in the case of tournaments is different. We would like to stress the fact that Definition 2 does not require all directed distances to be defined (not even the directed distances to all vertices in the resolving set), but only the distances to the vertices which resolve each pair of distinct vertices. 
Proposition 3. For any order $n$ tournament $T, \operatorname{Dim}(T) \leqslant n-1$.

Proof. It is well known that every tournament has a Hamiltonian path [15]. Suppose, then, that $u_{1} \ldots u_{n}$ is a Hamiltonian path for a tournament $T$. Then, given two vertices $u_{i}, u_{j}$ such that $i<j$, they can be resolved by $u_{j}$ since $d\left(u_{i}, u_{j}\right)>d\left(u_{j}, u_{j}\right)=0$. Therefore, $\left\{u_{2}, \ldots, u_{n}\right\}$ is a resolving set.

We observe that the upper bound of Proposition 3 is best possible for general tournaments.

Proposition 4. For every integer $n, \operatorname{Dim}\left(T T_{n}\right)=n-1$.

Proof. Let $V\left(T T_{n}\right)=\{1, \ldots, n\}$ be the numbering of the vertices from the definition of $T T_{n}$ given in Subsection 1.1 (that is, every arc ij satisfies $i<j$ ), and let $S$ be a determining set for $T T_{n}$. Then, given two vertices $i, j$ such that $i<j$, we have that

- vertex $j$ resolves the pair since $d(i, j)=1$ and $d(j, j)=0$,

- no vertex $k<j$ can resolve it since distance $d(j, k)$ is undefined, and

- no vertex $k>j$ can resolve it neither since $d(i, k)=d(j, k)=1$.

We conclude that $j$ is the only vertex which resolves a pair $i, j$ such that $i<j$, and then, must belong to $S$. Therefore, the only vertex that can be left out of $S$ is 1 , and we have $\operatorname{Dim}\left(T T_{n}\right) \geqslant n-1$. On the other hand, $\operatorname{Dim}\left(T T_{n}\right) \leqslant n-1$ by Proposition 3 , and the result is proved.

To establish the relation between resolving and determining sets, we just follow the proof by Erwin and Harary ([8], Lemma 2), which can be applied without changes to digraphs and we reproduce here for completeness.

Proposition 5. Every resolving set for a digraph is also a determining set. Therefore, for any digraph $D, \operatorname{Det}(D) \leqslant \operatorname{Dim}(D)$.

Proof. Let $S$ be a resolving set for a digraph $D$. To get a contradiction, suppose that $S$ is not a determining set for $D$ and, then, $\operatorname{Stab}(S) \neq S_{1}$. In this case, there must be a vertex $v$ and an automorphism $\phi \in \operatorname{Stab}(S)$ such that $v \neq \phi(v)$. Since $S$ is a resolving set for $D$, there must be a vertex $u \in S$ for which $d(v, u) \neq d(\phi(v), u)$. However,

$$
d(v, u)=d(\phi(v), \phi(u))=d(\phi(v), u)
$$

where the first equality holds because $\phi$ is an isometry, and the second one because $\phi$ fixes $u$, and we have a contradiction. 


\subsection{Outline}

In Section 2, we prove that the determining number of every tournament of order $n$ is tightly bounded by $\lfloor n / 3\rfloor$.

Section 3 is devoted to the metric dimension. We derive a tight upper bound of $\lfloor n / 2\rfloor$ in the case of strong tournaments of order $n$ (as we have just seen, there is no constant fraction upper bound in the general case). In order to prove this bound, we first answer a conjecture posed by Kannan, Naor, and Rudich on their concept of anchor ([12]), and link it to the metric dimension. We also show in Section 3 that resolving sets and anchors are exactly the same concept in regular tournaments.

\section{The Determining Number}

The determining number is 0 , for exemple, for the transitive tournament of $k$ vertices, $T T_{k}$, (in fact, for any rigid tournament) but how large can it be for a general tournament?

Proposition 6. For every $n>0$, there is an order $n$ tournament $T_{n}$ such that $\operatorname{Det}\left(T_{n}\right) \geqslant$ $\lfloor n / 3\rfloor$.

Proof. If $n=3 k$ for some integer $k$, let $T_{n}$ be the order $n$ tournament obtained from any tournament of order $k$ where each vertex has been subtituted by a copy of $C_{3}$. Observe that any determining set of cardinality smaller than $k$ would fail to contain all three vertices from at least one of the copies of $C_{3}$, and that would make it possible to rotate it independently of the rest of the vertices. Therefore, the determining number of $T_{n}$ must be at least $k$.

If $n \equiv d$ modulo 3 with $d=1$ or $d=2$, we take $T_{n}$ as the tournament $T_{n-d}$ defined above plus $d$ new vertices pointing to all vertices in $T_{n-d}$. Since the new vertices must be fixed in any nontrivial automorphism of $T_{n}$, $\operatorname{Det}\left(T_{n}\right)=\operatorname{Det}\left(T_{n-d}\right) \geqslant(n-d) / 3=$ $\lfloor n / 3\rfloor$.

Now we will see that there is always a determining set with at most $\lfloor n / 3\rfloor$ vertices for an $n$ order tournament. In the proof, we exploit the fact that an automorphism cannot move vertices between different orbits and, then, in order to construct a determining set for a tournament, it is enough to construct determining sets for the subtournaments induced by its orbits and then merge them. We will need the following proposition for digraphs, which is the direct translation of a similar one stated in [4] for graphs and does not require a separate proof.

Proposition 7. Let $\mathcal{O}_{1}, \ldots, \mathcal{O}_{k}$ be the vertex orbits of a digraph $D$. Let $H_{1}, \ldots, H_{k}$ be the associated induced subtournaments. Let $S_{1}, \ldots S_{k}$ be determining sets for $H_{1}, \ldots, H_{k}$. Then, $S=S_{1} \cup \cdots \cup S_{k}$ is a determining set for $D$.

Now, we can state the main theorem of this section.

Theorem 8. For every order $n$ tournament $T$, $\operatorname{Det}(T) \leqslant\lfloor n / 3\rfloor$. 
Proof. Given a tournament $T$ of order $n$, we will show that the subtournament $T[\mathcal{O}]$ induced by an orbit $\mathcal{O}$ of $T$ has a determining set $S_{\mathcal{O}}$ of size at most $\lfloor|\mathcal{O}| / 3\rfloor$. Therefore, the union of all determining sets for the different orbits $S=\bigcup_{\mathcal{O}}$ is an orbit of $T S_{\mathcal{O}}$ will satisfy that $|S| \leqslant\lfloor n / 3\rfloor$ while, by Proposition $7, S$ is a determining set for $T$. Thus, it is enough to prove the statement separately for each orbit.

Suppose then that $\mathcal{O}$ is an orbit of $T$, and that $|\mathcal{O}|=m$. Let $H=T[\mathcal{O}]$ be the subtournament of $T$ induced by $\mathcal{O}$. The fact that $\mathcal{O}$ is an orbit implies that $H$ must be vertex transitive (there exists $\phi \in \Gamma(H)$ such that $\phi(u)=v$ for any $u, v \in H$ ) and, hence, regular.

We will proceed by induction on $m$. For $m \leqslant 2$, the determining set can be empty since there is no nontrivial automorphism. For the inductive step, we will suppose that $m \geqslant 3$, but note that in the case $m=3$, the only tournaments are $T T_{3}$ (which is already rigid) and $C_{3}$ (which can be made rigid by fixing one vertex). Now, for $m \geqslant 3$, we consider three cases depending on the remainder of $m$ when dividing by 3 . All congruencies in the rest of the proof are taken modulo 3 .

Case 1: $m \equiv 0$.

Let $u$ be any vertex in $H$. Since $H$ is regular, $\left|H_{\rightarrow u}\right|=\left|H_{u \rightarrow}\right|$, which together with the fact that $\left|H_{\rightarrow u}\right|+\left|H_{u \rightarrow}\right|+1=m \equiv 0$ leaves the only possibility that $\left|H_{\rightarrow u}\right|=\left|H_{u \rightarrow}\right| \equiv 1$.

Therefore, there exists an integer $r$ such that $\left|H_{\rightarrow u}\right|=\left|H_{u \rightarrow}\right|=3 r+1$. By induction hypothesis, both $H_{\rightarrow u}$ and $H_{u \rightarrow}$ have determining sets of size $r$. Their union plus vertex $u$ form a determining set for $H$ of size $2 r+1$. Since $|H|=m=2(3 r+1)+1=6 r+3$, the determining set has at most (in this case, exactly) $\lfloor m / 3\rfloor$ vertices as expected.

Case 2: $m \equiv 2$.

Let $u$ be any vertex in $H$. As in the previous case, $\left|H_{\rightarrow u}\right|=\left|H_{u \rightarrow}\right|$, which now implies that $\left|H_{\rightarrow u}\right|=\left|H_{u \rightarrow}\right| \equiv 2$. Therefore, there exists an integer $r$ such that $\left|H_{\rightarrow u}\right|=$ $\left|H_{u \rightarrow}\right|=3 r+2$. By induction hypothesis, both $H_{\rightarrow u}$ and $H_{u \rightarrow}$ have determining sets of size $r$. Their union plus vertex $u$ form a determining set for $H$ of size $2 r+1$. Since $|H|=m=2(3 r+2)+1=6 r+5$, the determining set has at most (in this case, less than) $\lfloor m / 3\rfloor$ vertices.

Case 3: $m \equiv 1$.

In this case, for any $u \in H,\left|H_{\rightarrow u}\right|=\left|H_{u \rightarrow}\right| \equiv 0$. A determining set for $H$ constructed inductively as in the previous cases would have more than $\lfloor m / 3\rfloor$ vertices. However, we can complete the proof using two subcases:

- Subcase 3.1: For every arc uv $\in H,\left|H_{u \rightarrow v}\right| \equiv 2$.

Since we are assuming that $m \geqslant 3$, let $u, v, w$ be three vertices in $H$. Moreover, we can select them in such a way that $u v, v w$, and $w u$ are in $H$, the reason being that $H$ cannot be transitive in this subcase and, therefore, it must contain a 3-cycle. Now, $H$ can be split into the following subsets:

- $H_{u \rightarrow v}, H_{v \rightarrow w}$, and $H_{w \rightarrow u}$, whose cardinalities are all congruent with 2 modulo 
3. Let $\left|H_{u \rightarrow v}\right|=3 r+2,\left|H_{v \rightarrow w}\right|=3 s+2$, and $\left|H_{w \rightarrow u}\right|=3 t+2$ for some $r, s, t \geqslant 0$,

- the set $\{u, v, w\}$, and

- the remaining vertices in $H$, say $H^{\prime}$. Note that since $m \equiv 1$ and the number of vertices counted so far is multiple of $3,\left|H^{\prime}\right| \equiv 1$, say $\left|H^{\prime}\right|=3 z+1$ for some $z$.

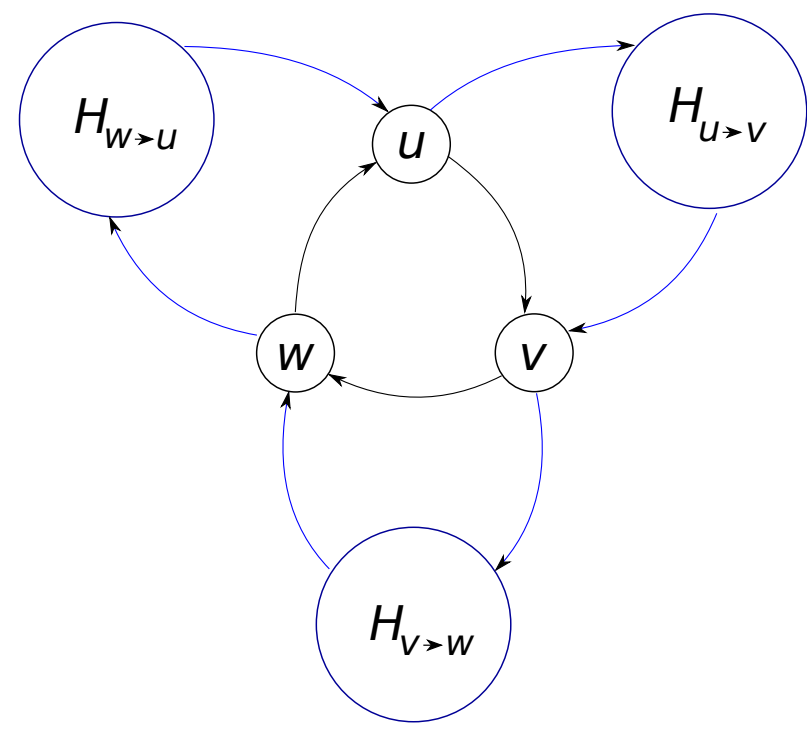

Figure 1: Subcase 3.1: $\left|H_{u \rightarrow v}\right| \equiv\left|H_{v \rightarrow w}\right| \equiv\left|H_{w \rightarrow u}\right| \equiv 2$.

Summing up the previous numbers, we have:

$$
\begin{aligned}
& m=\left|H_{u \rightarrow v}\right|+\left|H_{v \rightarrow w}\right|+\left|H_{w \rightarrow u}\right|+|\{u, v, w\}|+\left|H^{\prime}\right|= \\
& =(3 r+2)+(3 s+2)+(3 t+2)+3+(3 z+1)= \\
& =3(r+s+t+z)+10 .
\end{aligned}
$$

On the other hand, we can assume the existence of determining sets for $H_{u \rightarrow v}, H_{v \rightarrow w}$, $H_{w \rightarrow u}$, and $H^{\prime}$ by induction hypothesis, of sizes $r, s, t$, and $z$, respectively. Their union plus the vertices $u, v$, and $w$ is a determining set for $H$ with less than $\lfloor m / 3\rfloor$ vertices.

- Subcase 3.2: There is an arc uv $\in H$ for which $\left|H_{u \rightarrow v}\right| \not \equiv 2$.

Let $u, v$ be two such vertices. Now, we consider the partition of $H$ into the sets: $H_{u \rightarrow v}, H_{\rightarrow u v}, H_{v \rightarrow u}, H_{u v \rightarrow}$, and $\{u, v\}$. Using the assumption (of Case 3 ) that for any $w \in H,\left|H_{\rightarrow w}\right|=\left|H_{w \rightarrow}\right| \equiv 0$, we can observe the following:

i. $\left|H_{u \rightarrow v} \cup H_{\rightarrow u v}\right| \equiv 2$, since the previous union plus $v$ is exactly $H_{\rightarrow v}$.

ii. $\left|H_{\rightarrow u v} \cup H_{v \rightarrow u}\right| \equiv 0$, since the previous union is exactly $H_{v \rightarrow \text {. }}$.

iii. $\left|H_{v \rightarrow u} \cup H_{u v \rightarrow}\right| \equiv 2$, since the previous union plus $v$ is exactly $H_{u \rightarrow}$.

Now, we can see that there are only two possibilities: 


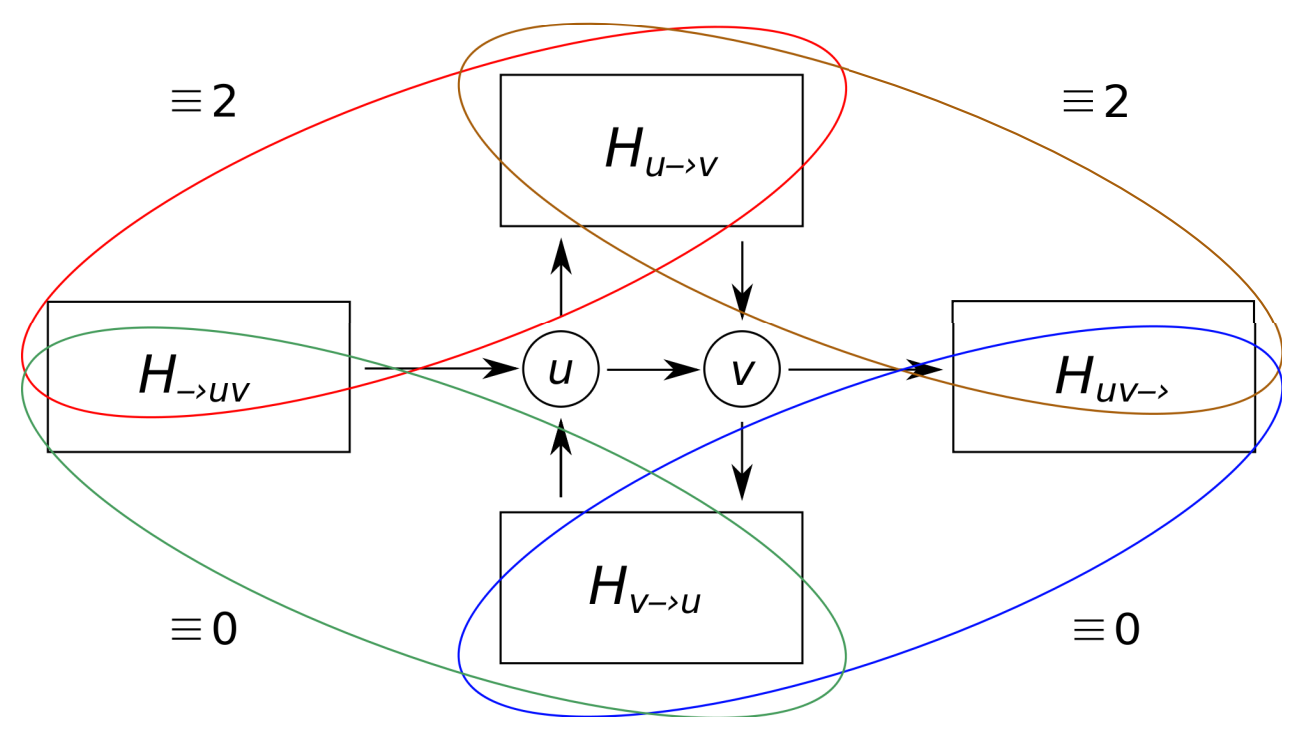

Figure 2: Subcase 3.2: For some arc $u v \in H, H_{u \rightarrow v} \mid \not \equiv 2$.

$-\left|H_{u \rightarrow v}\right| \equiv 1$. Then, by i, $\left|H_{\rightarrow u v}\right| \equiv 1$; by ii, $\left|H_{v \rightarrow u}\right| \equiv 2$; and by iii, $\left|H_{u v \rightarrow}\right| \equiv 1$. There must be some $r, s, t, z$ in this case such that $\left|H_{u \rightarrow v}\right|=3 r+1,\left|H_{\rightarrow u v}\right|=$ $3 s+1,\left|H_{v \rightarrow u}\right|=3 t+2$, and $\left|H_{u v \rightarrow}\right|=3 z+1$.

$-\left|H_{u \rightarrow v}\right| \equiv 0$. Then, by i, $\left|H_{\rightarrow u v}\right| \equiv 2$; by ii, $\left|H_{v \rightarrow u}\right| \equiv 1$; and by iii, $\left|H_{u v \rightarrow}\right| \equiv 2$. There must be som $r, s, t, z$ in this case such that $\left|H_{u \rightarrow v}\right|=3 r,\left|H_{\rightarrow u v}\right|=3 s+2$, $\left|H_{v \rightarrow u}\right|=3 t+1$, and $\left|H_{u v \rightarrow}\right|=3 z+2$.

In any of the above two possibilities, $H$ contains the four previous subsets plus $u$ and $v$, giving $|H|=3(r+s+t+z)+7$. The union of the determining sets given by the induction hypothesis plus $u$ and $v$ gives a determining set for $H$ of size $r+s+t+z+2$, which is less than $\lfloor m / 3\rfloor$.

Note that the upper bound $\lfloor n / 3\rfloor$ given in Theorem 8 is tight by Proposition 6 .

\section{The Metric Dimension}

As we have seen in Section 1, if we do not consider any additional condition on a tournament of order $n$, its metric dimension can be as large as $n-1$. In this section, we show that just requiring a tournament to be strong, its metric dimension drops to at most $\lfloor n / 2\rfloor$.

We start with the observation that some strong tournaments of order $n$ have metric dimension at least $\lfloor n / 2\rfloor$.

Proposition 9. For $n=3$ and for all $n \geqslant 5$, there is an order $n$ strong tournament $T_{n}$ such that $\operatorname{Dim}\left(T_{n}\right) \geqslant\lfloor n / 2\rfloor$. 
Proof. For $n=3$, there is only one strong tournament up to isomorphism and its metric dimension is 1 . Suppose, then, that $n \geqslant 5$.

If $n$ is even, let $k \geqslant 3$ be such that $n=2 k$ and let $T_{n}$ be the order $n$ tournament obtained from any strong tournament of order $k$ where each vertex has been substituted by a copy of $T T_{2}$ (each arc in the initial tournament generates four arcs in $T_{n}$ ). Observe that $T_{n}$ is strong and, furthermore, any resolving set for $T_{n}$ with cardinality smaller than $k$ would fail to contain the two vertices from one of the copies of $T T_{2}$ and then no vertex in the resolving set would be able to resolve them.

If $n$ is odd, let $k \geqslant 3$ be such that $n=2 k-1$. We construct $T_{n}$ from any strong tournament of order $k$ by duplicating $k-1$ of its vertices (as before, we substitute each vertex by $T T_{2}$ ). Again, $T_{n}$ must be strong and any resolving set for $T_{n}$ must contain at least one of the duplicated vertices, that is, a total of $k-1$ vertices.

In any of the two above cases, we have that $\operatorname{Dim}\left(T_{n}\right) \geqslant\lfloor n / 2\rfloor$.

Kannan, Naor, and Rudich [12] introduced the notion of anchors for tournaments in order to get a simple algorithm for tournament isomorphism. Anchors can be seen as a sort of simplification of resolving sets having the advantage that they are always defined.

An anchor in a tournament $T$ is a subset $S \subseteq V(T)$ such that for all vertices $u, v \in$ $V(T)-S, u \neq v$, there exists a vertex $w \in S$ such that exactly one of $u w$ and $v w$ is an arc of $T$, in which case we say that $w$ distinguishes the pair $u, v$. Thus, if the vertices in an anchor are fixed, all vertices in the tournament would be fixed and no automorphism other than the identity would be possible; in this respect, the concept is similar to the other symmetry breaking concepts seen so far. Let Anchor $(T)$ denote the size of the smallest anchor for $T$.

In [12], it is shown that for any order $n$ tournament $T$, Anchor $(T) \leqslant\lfloor 2 n / 3\rfloor$, and the authors conjecture that, in fact, $\operatorname{Anchor}(T) \leqslant\lfloor n / 2\rfloor$. We start proving here that their conjecture is correct.

Theorem 10. For every order $n$ tournament $T$, Anchor $(T) \leqslant\lfloor n / 2\rfloor$.

Proof. Let $T$ be a tournament with $n$ vertices. We will preceed by induction on $n$. The statement is trivially true for $n \leqslant 2$ by the definition of anchor. Now, suppose that $n \geqslant 3$ and consider two cases depending on the parity of $n$ :

Case 1: $n$ is even.

Select a vertex $u$ from $T$. Now, $V(T)-\{u\}$ can be split into the disjoint sets of vertices $T_{\rightarrow u}$ and $T_{u \rightarrow}$. Since $|V(T)-\{u\}|$ is odd, one of $\left|T_{\rightarrow u}\right|$ and $\left|T_{u \rightarrow}\right|$ is even and one is odd. Suppose without loss of generality that the first quantity is odd and equal to $2 r+1$ and the second one is even and equal to $2 s$, for integers $r, s$. Then, by induction hypothesis, $T_{\rightarrow u}$ has an anchor $A$ of size $r$, and $T_{u \rightarrow}$ has an anchor $B$ of size $s$.

Note that the set of vertices $S=A \cup B \cup\{u\}$ has size $r+s+1=n / 2$. Furthermore, $S$ is an anchor since for any $x, y \notin S$, we can consider three possibilities:

- $x, y \in T_{\rightarrow u}$. In this case, $x$ and $y$ are distinguished by some vertex in $A$.

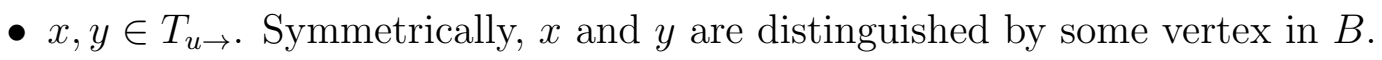




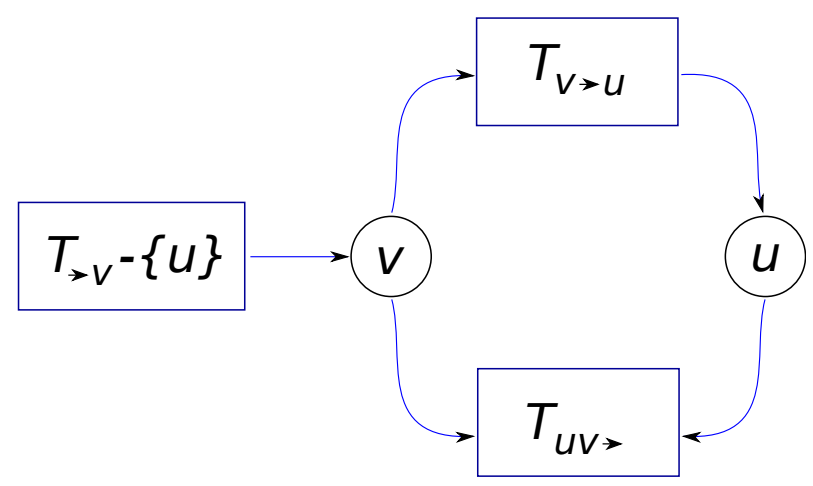

Figure 3: Subcase 2.1: $\left|T_{u v \rightarrow}\right|,\left|T_{v \rightarrow u}\right|$, and $\left|T_{\rightarrow v}-\{u\}\right|$ are odd.

- $x \in T_{u \rightarrow}$ and $y \in T_{\rightarrow u}$ or viceversa. In this case, vertex $u$ distinguishes $x$ and $y$.

\section{Case 2: $n$ is odd.}

Consider the parity of the sets $T_{u \rightarrow}$, for $u \in V$. If some of these sets has odd cardinality, then the corresponding set $T_{\rightarrow u}$ must have odd cardinality too; suppose the respective cardinalities are $2 r+1$ and $2 s+1$ for some integers $r, s$. By induction hypothesis, these two sets of vertices must have anchors of sizes $r$ and $s$, respectively. Similarly to the previous case, the union of the anchors plus vertex $u$ form an anchor for $T$ of size $r+s+1$. Note that, in this case, $n=(2 r+1)+(2 s+1)+1=2(r+s+1)+1$, so the anchor has size $\lfloor n / 2\rfloor$, as required.

Consider now the case in which all sets $T_{u \rightarrow}$, for $u \in V$, have even cardinality. Now, we consider two subcases:

- Subcase 2.1: There are two distinct vertices $u, v$ in $T$ s.t. $\left|T_{u v \rightarrow}\right|$ is odd.

We can assume, without loss of generality, that $u v \in T$. We can now split the set of vertices $T_{v \rightarrow}$ into two sets:

$$
T_{v \rightarrow}=T_{v \rightarrow u} \cup T_{u v \rightarrow}
$$

Since the above union is disjoint, $\left|T_{u v \rightarrow}\right|$ is odd, and $\left|T_{v \rightarrow}\right|$ is even, then $\left|T_{v \rightarrow u}\right|$ must be odd. We can classify all the vertices in $T$ different from $u$ and $v$ into the disjoint sets $T_{\rightarrow v}-\{u\}, T_{v \rightarrow u}$, and $T_{u v \rightarrow \text {. }}$

Since the last two sets have odd cardinality and the union of all three sets has cardinality $n-2$, which is odd, the first one must have odd cardinality too. Suppose that, for some integers $r, s$, and $t$,

$$
\left|T_{\rightarrow v}-\{u\}\right|=2 r+1,\left|T_{v \rightarrow u}\right|=2 s+1,\left|T_{u v \rightarrow}\right|=2 t+1 .
$$

By induction hypothesis, $T_{\rightarrow v}-\{u\}, T_{v \rightarrow u}$, and $T_{u v \rightarrow}$ must have anchors of respective sizes $r, s$, and $t$ which we will call $A, B$, and $C$, resp. Note that the set $S=A \cup B \cup$ $C \cup\{u, v\}$ contains $r+s+t+2$ vertices. Since $n=2(r+s+t)+5=2(r+s+t+2)+1$, 
$|S|$ is exactly $\lfloor n / 2\rfloor$. Now, we will argue that $S$ is an anchor for $T$. Given $x, y \notin S$, we have the following possibilities for $x$ and $y$ :

- In the case that both $x$ and $y$ belong to one of the sets $T_{\rightarrow v}-\{u\}, T_{v \rightarrow u}$, or $T_{u v \rightarrow}$, they can be distinguished by some vertex in the respective anchor: $A$, $B$, or $C$.

- If $x$ or $y$ is in $T_{\rightarrow v}-\{u\}$ and the other one is in either $T_{v \rightarrow u}$ or $T_{u v \rightarrow \text {, they are }}$ distinguished by $v$.

- If $x$ or $y$ is in $T_{v \rightarrow u}$ and the other one in $T_{u v \rightarrow}$, they are distinguished by $u$.

- Subcase 2.2: For all distinct vertices $u, v$ in $T,\left|T_{u v \rightarrow}\right|$ is even.

Note that, in this case, the tournament $T$ cannot be transitive since we can always select two "consecutive" vertices $u, v$ in a transitive tournament such that $\left|T_{u v \rightarrow}\right|$ is odd. Then, $T$ must contain a 3 -cycle, similarly to Subcase 3.1 in the proof of Theorem 8.

Let $u, v$, and $w$ be three vertices such that $u v, v w$, and $w u$ are three $\operatorname{arcs}$ in $T$. Note that we can partition the vertices in $T_{u \rightarrow}, T_{v \rightarrow}$, and $T_{w \rightarrow}$ as follows:

$$
\begin{aligned}
& T_{u \rightarrow}=T_{u \rightarrow v} \cup T_{u v \rightarrow} \cup\{v\} \\
& T_{v \rightarrow}=T_{v \rightarrow w} \cup T_{v w \rightarrow} \cup\{w\} \\
& T_{w \rightarrow}=T_{w \rightarrow u} \cup T_{w u \rightarrow} \cup\{u\}
\end{aligned}
$$

Our assumptions on cardinalities in this subcase applied to the above relations imply that the cardinalities of the sets $T_{u \rightarrow v}, T_{v \rightarrow w}$, and $T_{w \rightarrow u}$ must be odd, say

$$
\left|T_{u \rightarrow v}\right|=2 r+1, \quad\left|T_{v \rightarrow w}\right|=2 s+1, \text { and }\left|T_{w \rightarrow u}\right|=2 t+1
$$

for some integers $r, s$, and $t$. Note too that all three sets are disjoint. We now apply the induction hypothesis to the above sets and get an anchor $A$ for $T_{u \rightarrow v}$ with size $r$, an anchor $B$ for $T_{v \rightarrow w}$ with size $s$, and an anchor $C$ for $T_{w \rightarrow u}$ with size $t$. Let now $T^{\prime}$ be the set containing the rest of the vertices, that is

$$
T^{\prime}=V(T)-\left(T_{u \rightarrow v} \cup T_{v \rightarrow w} \cup T_{w \rightarrow u} \cup\{u, v, w\}\right) .
$$

The set $T^{\prime}$ must have odd cardinality, say $\left|T^{\prime}\right|=2 m+1$, and (by induction hypothesis) an anchor $D$ of size $m$.

Consider the set $S=A \cup B \cup C \cup D \cup\{u, v, w\}$. Its size is $r+s+t+m+3$, whereas $n=2(r+s+t+m)+7=2(r+s+t+m+3)+1$; therefore, $|S|=\lfloor n / 2\rfloor$. Now, we will argue that $S$ constitutes an anchor for $T$. Given $x, y \notin S$, we have the following possibilities for $x$ and $y$ :

- In the case that both $x$ and $y$ belong to one of the sets $T_{u \rightarrow v}, T_{v \rightarrow w}, T_{w \rightarrow u}$, or $T^{\prime}$, they can be distinguished by some vertex in the respective anchor: $A, B$, $C$, or $D$. 


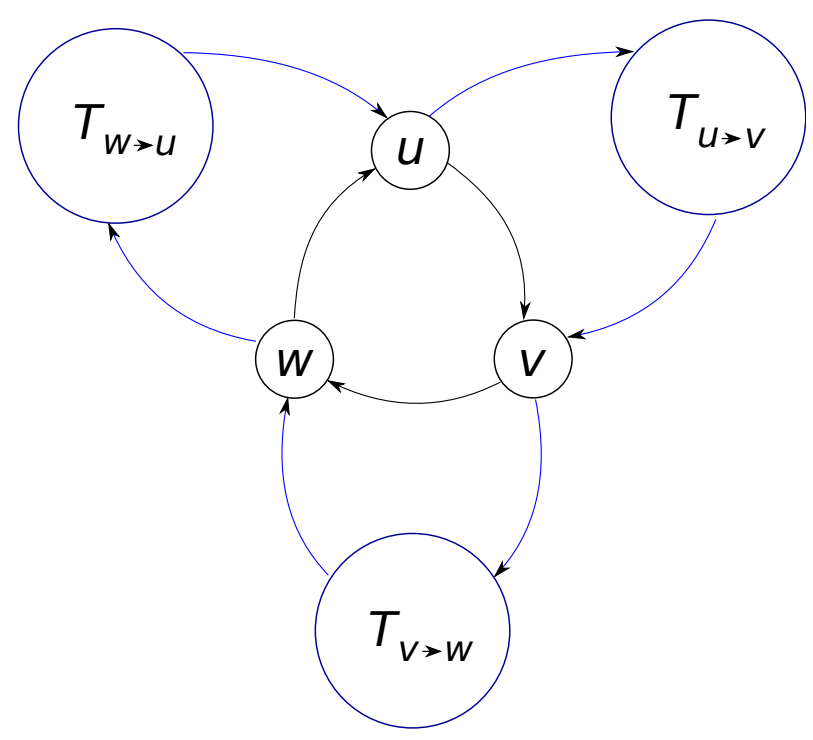

Figure 4: Subcase 2.2: $\left|T_{u \rightarrow v}\right|,\left|T_{v \rightarrow w}\right|$, and $\left|T_{w \rightarrow u}\right|$ are odd.

- In the case that $x$ and $y$ belong to different sets from $T_{u \rightarrow v}, T_{v \rightarrow w}, T_{w \rightarrow u}$, then $u, v$, or $w$ must distinguish $x$ and $y$. For example, if $x \in T_{u \rightarrow v}$ and $y \in T_{v \rightarrow w}$, they are distinguished by $v$, the rest of the cases being similar.

- In the case that one of $x, y$ is in $T^{\prime}$ and the other one is in $T_{u \rightarrow v}, T_{v \rightarrow w}$, or $T_{w \rightarrow u}$, then $u, v$, or $w$ will distinguish $x$ and $y$. For example, suppose that $x \in T^{\prime}$ and $y \in T_{u \rightarrow v}$. Since vertices in $T^{\prime}$ are not in $y \in T_{u \rightarrow v}$, it means that either $u x$ or $x v$ cannot be an arc in $T$, which means that either $x u$ or $v x$ is an arch in $T$ : in the first case, $x$ and $y$ are distinguished by $u$, in the second one, by $v$. The rest of the cases are similar.

The previous upper bound for the size of anchors has some implications regarding the metric dimension. In the first place, we note the following relation between the two notions.

Proposition 11. Every anchor in a strong tournament is a resolving set.

Proof. Suppose that $S$ is an anchor in a strong tournament $T$, and take two distinct vertices $u, v \in V(T)-S$ which are distinguished by a vertex $w \in S$. According to the definition of anchor, $T$ contains exactly one of the arcs $u w$ and $v w$. If it contains $u w$ (and then, $w v)$, we have $d(u, w)=1$ while $d(v, w)$ exists (because $T$ is strong) but must be greater than 1 . Then, $d(v, w)>d(u, w)$. The case when $T$ contains the arc $v w$ but not $u w$ similarly implies that $d(v, w)<d(u, w)$. In either case, $d(u, w) \neq d(v, w)$, and so $w$ resolves $u$ and $v$. 
Since a resolving set for $T$ must resolve any pair $u, v$ of distinct vertices in $T$ (not only in $V(T)-S)$, suppose that at least one of them, say $u$, belongs to $S$. In this case, vertex $u$ itself resolves the pair since $d(u, u)=0<d(v, u)$.

Now, combining Theorem 10 with Proposition 11, we get our main conclusion on the metric dimension of tournaments.

Corollary 12. For every order $n$ strong tournament $T, \operatorname{Dim}(T) \leqslant\lfloor n / 2\rfloor$.

Note that the upper bound of $\lfloor n / 2\rfloor$ for the metric dimension of strong tournaments is tight by Proposition 9 for all $n \neq 4$.

It is interesting to observe that resolving sets and anchors become the same notion when applied to regular tournaments.

Proposition 13. Let $T$ be a regular tournament. Then:

1. Every anchor in $T$ is a resolving set.

2. Every resolving set in $T$ is an anchor.

Proof.

1. By the fact that regular tournaments are strong [1] and Proposition 11.

2. Alspach proved in [1] that each arc of a regular tournament of order $n$ is contained in a cycle of each length $k, k=3, \ldots, n$. Taking $k=3$, we can conclude any two distinct vertices $u, v$ in $T$ belong to a directed 3-cycle, and so we can assure that either $d(u, v)=1$ or $d(u, v)=2$. Then, a vertex $w$ in a resolving set for $T$ can only resolve two other vertices $u, v$ by keeping distance 1 to one of them and 2 to the other; this means that $T$ contains exactly one of the arcs $u w, v w$ and, then, $w$ distinguishes $u$ and $v$. Thus, any resolving set is, in fact, an anchor.

In the case of regular tournaments, we can also add a lower bound due to the fact that any anchor of an order $n$ tournament must have at least $\lceil\log n\rceil-1$ vertices [12]. Then, $\operatorname{Dim}(T) \geqslant\lceil\log n\rceil-1$ for every regular tournament $T$ of order $n$.

\section{Acknowledgements}

The motivation for this research started while the author was visiting the LRI at Université Paris Sud under an invitation of Miklos Santha. The author would like to thank Christoph Dürr for several helpful conversations on the topic of this paper and to an anonymous referee for contributing to improve the paper and, in particular, Propositions 6 and 9. 


\section{References}

[1] B. Alspach. Cycles of each length in regular tournaments. Canad. Math. Bull., 10:283-286, 1967.

[2] M. Albertson and D. Boutin. Using Determining Sets to Distinguish Kneser Graphs. Electronic Journal of Combinatorics, 14:R20, 2007.

[3] M. Albertson and K. Collins. Symmetry Breaking in Graphs. Electronic Journal of Combinatorics, 3:R18, 1996.

[4] D. Boutin. Identifying Graph Automorphisms Using Determining Sets. The Electronic Journal of Combinatorics, 13, \#R78, 2006.

[5] G. Chartrand, L. Eroh, M. Johnson, and O. Oellermann. Resolvability in graphs and the metric dimension of a graph. Discrete Applied Mathematics, 105:99-113, 2000.

[6] M. Chan. The distinguishing number of the augmented cube and hypercube powers. Discrete Mathematics, 308:2330-2336, 2008.

[7] G. Chartrand, M. Raines, and P. Zhang. The Directed Distance Dimension of Oriented Graphs. Mathematica Bohemica, 125(2):155-168, 2000.

[8] D. Erwin and F. Harary. Destroying Automorphisms by Fixing Nodes. Dicrete Mathematics, 306:3244-3252, 2006.

[9] F. Harary. Methods of Destroying the Symmetries of a Graph. Bull. Malaysian Math. Sci. Soc., 24(2):183-191, 2001.

[10] F. Harary and R.A. Melter. On the metric dimension of a graph. Ars Combinatorica, 2:191-195, 1976.

[11] F. Harary and L. Moser. The Theory of Round Robin Tournamnets. The American Mathematical Monthly, 73(3):231-246, 1966.

[12] S. Kannan, M. Naor, and S. Rudich. Anchors in Tournaments. Technical Report TR 92-08, Dept. of Computer Science, University of Arizona, 1992.

[13] S. Khuller, B. Raghavachari, and A. Rosenfeld. Landmarks in Graphs. Discrete Appl. Math., 70:217-229, 1996.

[14] J. W. Moon. Topics on Tournaments. Holt, Rinehart and Winston, New York, 1968.

[15] L. Redei. Ein kombinatoricher Satz. Acta Litt. Szeged, 7:39-43, 1934.

[16] P.J. Slater. Leaves of trees. Congr. Numer., 14:549-559, 1975.

[17] B. Shanmukha, B. Sooryanarayana, and K.S. Harinath. Metric Dimension of Wheels. Far East J. Appl. Math., 8(3):217-229, 2002. 